\title{
Editorial
}

Ophthalmic

\section{The 'Free-Floating' Retina Epoch: New Models for New Scopes}

\author{
Solon Thanos \\ Department of Experimental Ophthalmology, School of Medicine, University Eye Hospital Münster, \\ Münster, Germany
}

The retina, which is a tiny central nervous layer about $0.3 \mathrm{~mm}$ thick in human, has a highly complex function and a marvelous multilaminated structure that has inspired many studies over the last two centuries. One can review hundreds of articles published annually on the retina, each being focused on a specific scope ranging from anatomy to the more recently promoted disciplines of genomics, proteomics, pharmacology and cell biology. Taken together, all these studies continue to enrich and develop our knowledge of the retinal structure and function, with implications reaching far into the cerebral cortex which is thicker, but organized in a similar way, just reminding of being a large appendix of the retina.

Although it would be unjust to underestimate the works that have been performed in vivo or in cell cultures, cultures of the whole retinal tissue have also greatly contributed to the development of our knowledge. In particular, it was the innovative experiments by Hild and Callas [1] in 1967 that initialized a new era of retinal research by culturing retinal tissue in vitro and studying its cytoarchitecture. The later studies of this epoch that can be named the 'organotypic culture epoch' have indeed shed light into many aspects of retinal development, differentiation survival of cells, neuroglial interactions, growth factor activities, axonal guidance and axonal regeneration. While cultures may be either stationary flatmount cultures of embryonic to adult stages or free-float- ing cultures within rotating tubes, the scope of circumventing disadvantages of in vivo experimentation is clearly outlined. On the other hand, cultures of complex tissues which permit cellular interactions in a fashion mimicking the in vivo conditions are certainly more valuable than dissociated cell cultures or cell lines.

The paper published in the present issue by Rzeczinski and Victorov et al. [2] shows that the authors have succeeded in establishing the roller culture of free-floating adult retina, a procedure that may be suitable for other vertebrate retinas too. When viewed from the clinicalpathological standpoint, the model appears promising for examining diseases such as glaucoma, hereditary retinal dystrophies or proliferative diseases like diabetic retinopathy. Apart from potential use in human retinal research, the free-floating method provides the advantage that it can be used in pharmacological and/or toxicological studies as well, and save experiments on living animals, a socioethical demand on researchers. Last but not least, the roller culture method adds to the long-lasting efforts of providing relatively simple alternatives to cultures of dissociated cells. Although it would be unfair to not honor the numerous former models of retinal cultures, the scientific community should be indebted to the authors of the present study for clearly outlining a modification of retinal cultures and providing a new tool for certain purposes.

\section{KARGER}

Fax +4161306 1234

E-Mail karger@karger.ch

www.karger.com (c) 2006 S. Karger AG, Basel

0030-3747/06/0385-0261\$23.50/0

Accessible online at:

www.karger.com/ore
S. Thanos

Department of Experimental Ophthalmology

School of Medicine, University of Münster

Domagkstrasse 15, DE-48149 Münster (Germany)

Tel. +49 251835 6915, Fax +49251835 6916, E-Mail solon@uni-muenster.de 
The increase in our knowledge of the retina is instructive as it shows that each advance has been based on improvement of preexisting methods or development of new methods. The use of these methods in conscientious research may help in finding unsuspected facts or discovering new cellular responses to changes in the surround. We can thus welcome each new methodology and recognize how important the improvement of methods is for acquisition of knowledge.
References

1 Hild W, Callas G: The behavior of retinal tissue in vitro, light and electron microscopic observations. Z Zellforsch Mikrosk Anat 1967;80:1-21.

2 Rzeczinski S, Victorov IV, Lyjin AA, Aleksandrova OP, Harms C, Kronenberg G, Freyer D, Scheibe F, Priller J, Endres M, Dirnagel $\mathrm{U}$ : Roller culture of free-floating retinal slices: a new system of organotypic cultures of adult rat retina. Ophthalmic Res 2006;38: 263-269. 